\title{
RAF inhibitors transactivate RAF dimers and ERK signalling in cells with wild-type BRAF
}

\author{
Poulikos I. Poulikakos ${ }^{1}$, Chao Zhang ${ }^{2}$, Gideon Bollag ${ }^{3}$, Kevan M. Shokat ${ }^{2} \&$ Neal Rosen ${ }^{1}$
}

Tumours with mutant BRAF are dependent on the RAF-MEKERK signalling pathway for their growth ${ }^{1-3}$. We found that ATPcompetitive RAF inhibitors inhibit ERK signalling in cells with mutant BRAF, but unexpectedly enhance signalling in cells with wild-type BRAF. Here we demonstrate the mechanistic basis for these findings. We used chemical genetic methods to show that drugmediated transactivation of RAF dimers is responsible for paradoxical activation of the enzyme by inhibitors. Induction of ERK signalling requires direct binding of the drug to the ATP-binding site of one kinase of the dimer and is dependent on RAS activity. Drug binding to one member of RAF homodimers (CRAF-CRAF) or heterodimers (CRAF-BRAF) inhibits one protomer, but results in transactivation of the drug-free protomer. In BRAF(V600E) tumours, RAS is not activated, thus transactivation is minimal and ERK signalling is inhibited in cells exposed to RAF inhibitors. These results indicate that RAF inhibitors will be effective in tumours in which BRAF is mutated. Furthermore, because RAF inhibitors do not inhibit ERK signalling in other cells, the model predicts that they would have a higher therapeutic index and greater antitumour activity than mitogen-activated protein kinase (MEK) inhibitors, but could also cause toxicity due to MEK/ERK activation. These predictions have been borne out in a recent clinical trial of the RAF inhibitor PLX4032 (refs 4, 5). The model indicates that promotion of RAF dimerization by elevation of wild-type RAF expression or RAS activity could lead to drug resistance in mutant BRAF tumours. In agreement with this prediction, RAF inhibitors do not inhibit ERK signalling in cells that coexpress BRAF(V600E) and mutant RAS.

Six distinct ATP-competitive RAF inhibitors induced ERK activation in cells with wild-type BRAF but inhibited signalling in mutant BRAF(V600E) cells (Fig. 1a, b, Supplementary Fig. 2a, b and data not shown; structures of compounds are shown in Supplementary Fig. 3, except that of PLX4032, which is unavailable). PLX4720 (ref. 6), and its analogue in clinical trial PLX4032, were studied in more detail. PLX4032 inhibited ARAF, BRAF and CRAF immunoprecipitated from $293 \mathrm{H}$ cells (Supplementary Fig. 4) and purified catalytic domains of BRAF(V600E), wild-type BRAF and CRAF (half-maximum inhibitory concentration ( $\mathrm{IC}_{50}$ ) values of 35,110 and $48 \mathrm{nM}$, respectively) (Supplementary Table 1). PLX4032 was assayed against 62 additional kinases that span the kinome, and had $\mathrm{IC}_{50}$ values of $1-10 \mu \mathrm{M}$ against eight of these and greater than $10 \mu \mathrm{M}$ against the rest (G.B., unpublished data). Induction of ERK signalling by PLX4720 was rapid (Fig. 1c), reversible (Fig. 1d) and associated with increased phosphorylation of the ERK substrate RSK (Fig. 1b). MEK and ERK phosphorylation were induced at intermediate concentrations of RAF inhibitor, and inhibited at much higher doses (Fig. 1a).

Physiological induction of ERK signalling depends on upstream activation of RAS by receptor-induced signalling ${ }^{7,8}$. PLX4032 induced ERK signalling in SKBR3 breast cancer cells, in which RAS activation is
HER2 dependent ${ }^{9}$. The HER2 inhibitor lapatinib abolished basal and PLX4032-induced ERK signalling in these cells (Supplementary Fig. 5a). In $293 \mathrm{H}$ cells, induction of MEK and ERK phosphorylation by either PLX4032 or PLX4720 was barely detectable (referred to hereafter as PLX4032/PLX4720 to indicate data obtained with both compounds). Haemagglutinin (HA)-tagged wild-type RAS overexpression resulted in enhanced MEK/ERK activation by RAF inhibitor, which was more pronounced when mutant RAS was overexpressed (Fig. 2a and Supplementary Fig. 5b). The results indicate that RAS activity is required for MEK/ERK activation by RAF inhibitors. In contrast, in $293 \mathrm{H}$ cells expressing Flag-tagged BRAF(V600E), ERK signalling was inhibited by PLX4032 (Supplementary Fig. 5c). These results indicate that RAF inhibitors will inhibit the growth of tumours with mutant BRAF, but not those with wild-type BRAF, including those with RAS mutation. This is

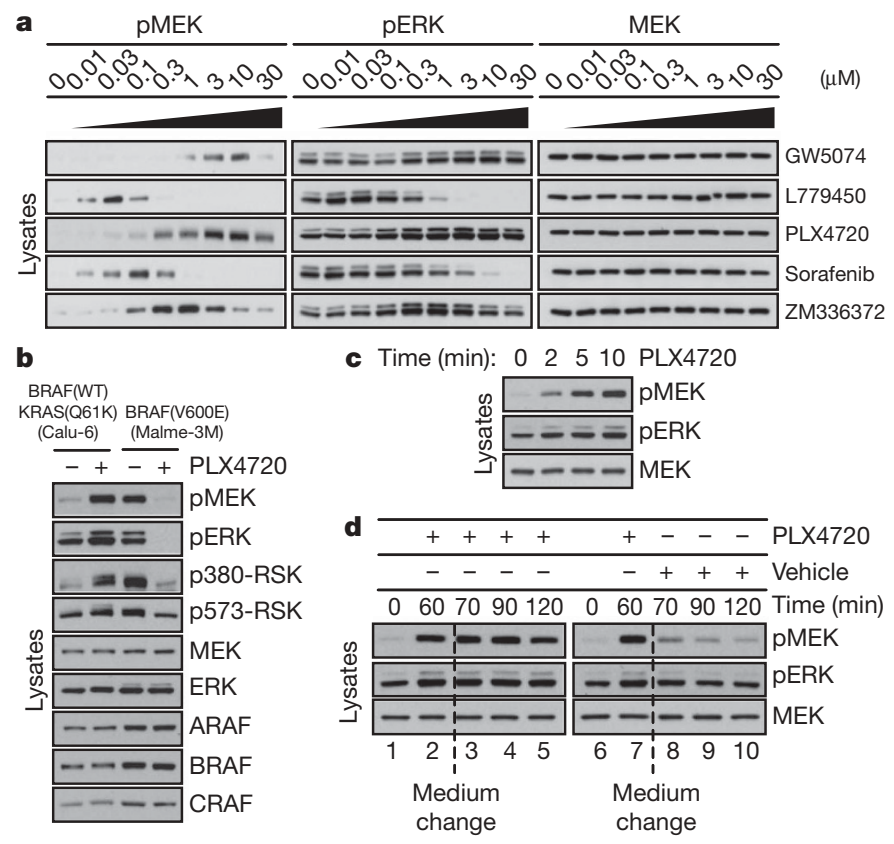

Figure 1 | RAF inhibitors rapidly activate MEK/ERK in cells with wild-type BRAF. a, Calu- 6 cells (wild-type BRAF $(\operatorname{BRAF}(\mathrm{WT})) / \mathrm{KRAS}(\mathrm{Q} 61 \mathrm{~K})$ ) were treated with increasing doses of the indicated RAF inhibitors and the effects on ERK signalling were determined by immunoblotting for phosphorylated MEK (pMEK) and phosphorylated ERK (pERK). b, Cells with wild-type BRAF (Calu-6) or mutant BRAF (Malme-3M) were treated with vehicle or PLX4720 $(1 \mu \mathrm{M}$ for $1 \mathrm{~h})$. Phosphorylation and expression of the indicated proteins were assayed by immunoblotting. c, Calu- 6 cells treated with $1 \mu$ M PLX 4720 for the indicated time points. d, Calu- 6 cells were treated with $1 \mu \mathrm{M}$ PLX4720 for $60 \mathrm{~min}$, then medium was replaced with medium containing $1 \mu \mathrm{M}$ PLX4720 (lanes 3-5) or vehicle (lanes 8-10) for the indicated time points. 


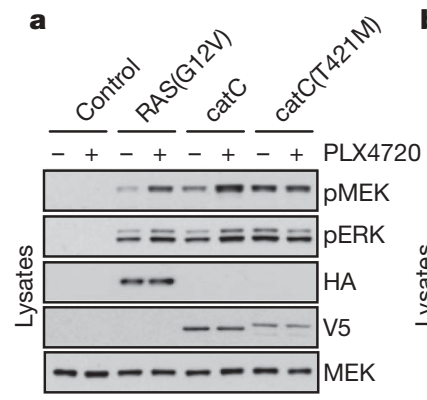

b

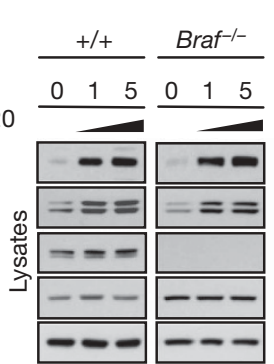

Figure 2 MEK/ERK activation requires binding of drug to the catalytic domain of RAF. a, 293H cells transfected with EGFP (control), HA-tagged RAS(G12V), the catalytic domain of CRAF (V5-tagged catC) and catC carrying a mutation at the gatekeeper residue (V5-tagged catC(T421M)), treated with vehicle or PLX4720 ( $1 \mu \mathrm{M}$ for $1 \mathrm{~h})$. Lysates were subjected to immunoblot analysis for pMEK and pERK. $\mathbf{b}$, Wild-type $(+/+)$, BRAF

indeed the case: MEK-dependent tumours with RAS mutation are unaffected by PLX4032 (N.R., unpublished data).

BRAF and CRAF kinases form homo- and heterodimers on RAS activation $^{10-12}$. PLX4032/PLX4720 induced pronounced phosphorylation of MEK and ERK in wild-type mouse embryonic fibroblasts (MEFs) and $\mathrm{Braf}^{-1-} \mathrm{MEFs}$. The response was diminished markedly in $\mathrm{Craf}^{-1-}$ (also called Raf1) MEFs (Fig. 2b and Supplementary Fig. 6a). Coexpression of CRAF and active RAS in $\mathrm{Craf}^{-1-}$ MEFs reconstituted the wild-type phenotype (Supplementary Fig. 6b, c). We conclude that BRAF is dispensable for MEK/ERK activation by PLX4032/PLX4720, and that CRAF expression is required for significant induction. We therefore investigated the mechanism of CRAF-dependent induction of ERK signalling in response to the drug.

Autoinhibition of RAF by its amino-terminal domain ${ }^{13}$ is relieved on binding to activated RAS ${ }^{7}$. We asked whether overexpression of an $\mathrm{N}$-truncated form of CRAF would bypass the requirement for RAS activity. In $293 \mathrm{H}$ cells expressing the catalytic domain of CRAF (catC), PLX4032/PLX4720 caused marked induction of MEK and ERK phosphorylation (Fig. 2a and Supplementary Fig. 7a). We focused mechanistic investigations on catC, in which PLX4032/PLX4720induced MEK/ERK activation is RAS independent. To test whether direct binding of PLX4032/PLX4720 to CRAF is required for induction of signalling, we generated a catC carrying a mutation at the gatekeeper position (T421) in the kinase domain (mutations used and their properties are in Supplementary Fig. 1a). Structural studies ${ }^{6}$ predict that the T421M mutation should prevent drug binding and catC(T421M) was indeed resistant to inhibition by PLX4032/PLX4720 in vitro (Supplementary Fig. 8a, b). ERK signalling was not induced by PLX4032/PLX4720 in cells expressing catC(T421M) (Fig. 2a and Supplementary Fig. 7b). Thus, activation of MEK/ERK by PLX4032/ PLX4720 depends on its direct binding to the RAF kinase active site. Sorafenib inhibited catC(T421M) in vitro (Supplementary Fig. 8c) and induced ERK signalling in cells expressing catC(T421M) (Fig. 2c), demonstrating that this mutant is capable of inhibitor-induced MEK/ERK activation. Thus, direct binding of an ATP-competitive inhibitor to CRAF is required for induction of ERK signalling.

Recent work shows that binding of ATP-competitive inhibitors to AKT and protein kinase $C$ inhibits their activity, but induces the active, phosphorylated state of these kinases ${ }^{14,15}$. Washed catC immunoprecipitated from PLX4032/PLX4720-treated cells was more active than that isolated from untreated cells (Fig. 3a and Supplementary Fig. 9a). The same was true for endogenous BRAF and CRAF immunoprecipitated from Calu-6 cells (Fig. $3 \mathrm{~b}$ and Supplementary Fig. 9b). Phosphorylation of CRAF at S338 and S621 has been correlated with its activation ${ }^{7}$. PLX4032/PLX4720 caused increased phosphorylation of both sites on wild-type and kinase-dead CRAF in $293 \mathrm{H}$ cells. In contrast, it did not affect the phosphorylation of the PLX4032/PLX4720-resistant CRAF(T421M) mutant (Fig. 3c and Supplementary Fig. 9c). All RAF inhibitors tested induced phosphorylation at $\mathrm{p} 338$ of endogenous CRAF

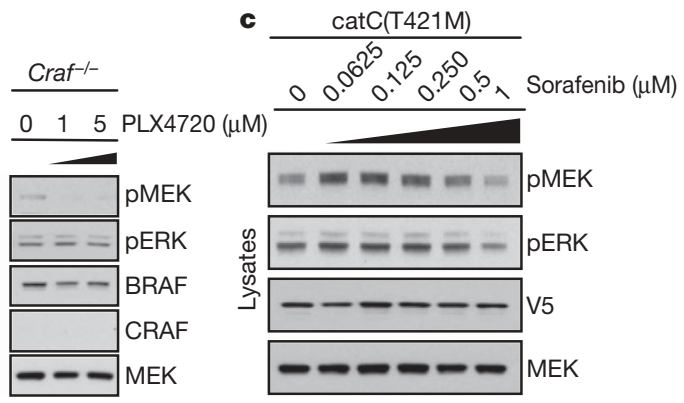

knockout $\left(\mathrm{Braf}^{-1-}\right)$ or CRAF knockout $\left(\mathrm{Craf}^{-1-}\right)$ MEFs were treated with the indicated concentrations of PLX4720 for $1 \mathrm{~h}$. c, Sorafenib inhibits the gatekeeper mutant catC(T421M) protein in vitro (Supplementary Fig. 8c) and activates MEK/ERK in cells expressing it. $293 \mathrm{H}$ cells overexpressing catC $(\mathrm{T} 421 \mathrm{M})$ were treated with the indicated concentrations of sorafenib for $1 \mathrm{~h}$. Lysates were subjected to analysis for pMEK and pERK.

(Fig. 3d). The data suggest that binding of PLX4032/PLX4720 to CRAF induces activation of the enzyme and, subsequently, ERK signalling. The result seems paradoxical: binding of ATP-competitive inhibitors to the catalytic domain of CRAF activates its function.

RAF isoforms form dimers in cells ${ }^{10-12,16}$. Because binding of both the drug and ATP to the catalytic domain would be required for activation and cannot occur simultaneously on the same molecule, we hypothesized that RAF inhibitors activate CRAF dimers in trans (Supplementary Fig. 1b). To test this model, we generated mutant catC(S428C) that binds to 6-acrylamido-4-anilinoquinazolines ${ }^{17}$, whereas catC does not. Two inhibitors, JAB13 (ref. 17) and JAB34 (also called PD-168393) ${ }^{18}$, both inhibited catC(S428C), but up to $30 \mu \mathrm{M}$ had no effect in vitro on catC (Supplementary Fig. 10a, b). $\mathrm{JAB} 13$ and JAB34 selectively affected ERK signalling in cells expressing catC(S428C) and were inactive in those expressing catC (Supplementary Fig. 11). Like the other inhibitors (Fig. 1a), lower doses ( $40 \mathrm{nM}$ to

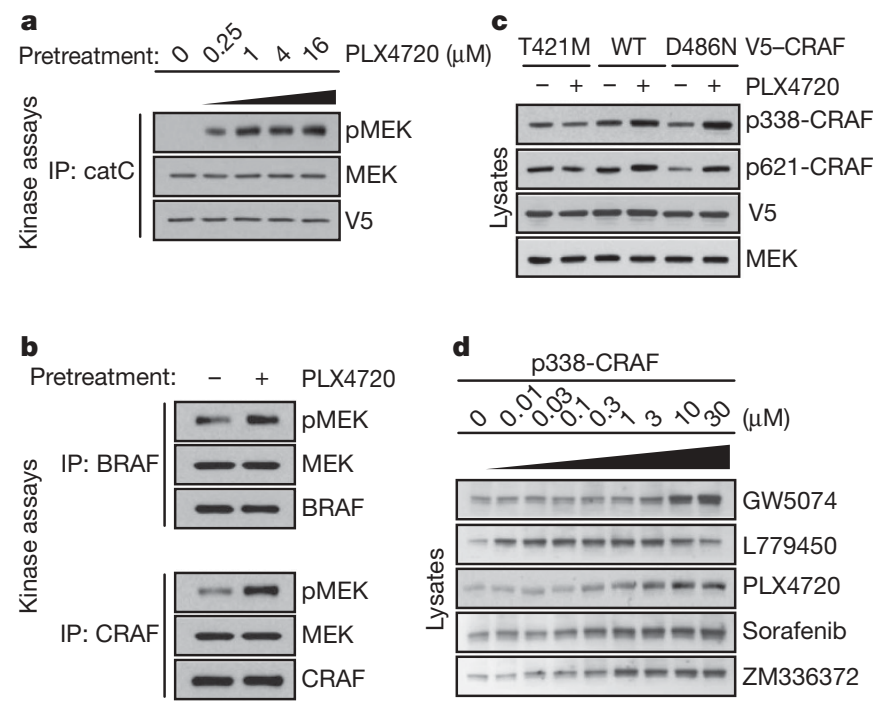

Figure 3 | RAF inhibitor induces the active, phosphorylated state of wildtype and kinase-dead RAF. a, $293 \mathrm{H}$ cells overexpressing cat $\mathrm{C}$ were treated with the indicated amounts of PLX4720 for $1 \mathrm{~h}$. Cells were lysed, catC was immunoprecipitated (IP), washed extensively and subjected to kinase assay. Kinase activity was determined by immunoblotting for pMEK. $\mathbf{b}$, Calu- 6 cells were treated with PLX4720 ( $1 \mu \mathrm{M}$ for $1 \mathrm{~h}$ ). Endogenous BRAF and CRAF were immunoprecipitated, washed and assayed for kinase activity. c, Treatment with RAF inhibitor results in elevated phosphorylation at activating phosphorylation sites on RAF. V5-tagged wild-type CRAF or kinase-dead CRAF (D486N) were overexpressed in $293 \mathrm{H}$ cells. After $24 \mathrm{~h}$ cells were treated with vehicle or PLX4720 ( $5 \mu \mathrm{M}$ for $1 \mathrm{~h}$ ) and lysates were immunoblotted for p338-CRAF and p621-CRAF. The gatekeeper mutant CRAF(T421M) was used as negative control. d, Samples as in Fig. 1a, immunoblotted for pS338CRAF. Note that phosphorylation at S338 steadily increased, even when concentrations were reached that inhibited MEK/ERK. 
$1 \mu \mathrm{M}$ ) induced ERK signalling (Supplementary Fig. 11), whereas higher doses $(10 \mu \mathrm{M})$ caused inhibition (Fig. 4a). The specificity of this system allows us to test the dimer transactivation model.

We coexpressed a V5-tagged, JAB-sensitive, kinase-dead catC, V5catC(S428C/D486N), and Flag-tagged catC in $293 \mathrm{H}$ cells. V5catC(S428C/D486N) is deficient in catalytic activity; it can bind to the inhibitor (JAB34) but cannot phosphorylate MEK, whereas FlagcatC is catalytically active, but cannot bind JAB34. Treatment of cells expressing both constructs with a concentration of JAB34 that inhibited ERK signalling in cells expressing catC(S428C) alone $(10 \mu \mathrm{M}$ JAB34, Fig. $4 \mathrm{a})$ resulted in marked induction of ERK signalling (Fig. 4b, lanes 5, 6). Thus, binding of JAB34 to kinase-dead, V5-catC(S428C/D486N) transactivated the catalytically competent Flag-catC. When the catalytically active drug-binding mutant V5catC(S428C) is coexpressed with catalytically inactive catC (FlagcatC(D486N)), $10 \mu \mathrm{M}$ JAB34 inhibited, rather than activated, ERK signalling (Fig. 4b, lanes 9, 10). When both constructs were insensitive to JAB, JAB34 had no effect on ERK signalling (Fig. 4b, lanes 1, 2). When both constructs were catalytically active, we observed moderate MEK/ERK activation, probably resulting from inhibition of V5catC(S428C) and transactivation of Flag-catC (Fig. 4b, lanes 3, 4).

Transactivation from CRAF to BRAF can occur as well. JAB34 activated ERK signalling in cells coexpressing Flag-BRAF and V5catC(S428C/D486N) (Fig. 4c). Finally, JAB34 induced ERK activation in cells coexpressing full-length V5-CRAF(S428C/D486N) and full-length Flag-CRAF, confirming that our model is valid in the context of full-length CRAF (Supplementary Fig. 12).

Thus, activation of RAF by ATP-competitive inhibitors can be explained by transactivation: binding of drug to one RAF in the dimer activates the other. This is consistent with the enhancement of induction by active RAS, which promotes homo- and heterodimerization of BRAF and CRAF ${ }^{10,12}$. Our model suggests that transactivation will be dependent on formation of RAF dimers. A side-to-side dimer of the kinase domain is observed in crystal structures of $\mathrm{BRAF}^{11}$, and the residues at the dimer interface are conserved in all RAF isoforms. On the basis of the BRAF crystal structures, we identified a conserved Arg (R509) at the centre of the dimer interface. Structural analysis predicts that mutation of R509 will diminish contacts between the two interacting proteins and reduce dimer formation, as also recently reported ${ }^{19}$. In that study, mutation of BRAF at R509 to histidine resulted in a marked loss of activity. The corresponding mutation in catC $(\mathrm{R} 401 \mathrm{H})$ results in severe loss of both expression and activity (data not shown). We therefore mutated R401 to alanine in V5-catC(S428C) and FlagcatC. This mutation diminished dimerization (Supplementary Fig. 13) but retained expression and activity. In cells coexpressing these mutants, JAB34 failed to induce ERK signalling (Fig. 4b, lanes 7, 8). Thus, a mutation that affects dimerization prevents transactivation.

The transactivation model explains the observation that inhibitors of RAF activate ERK signalling at low concentrations, but inhibit at higher concentrations in wild-type BRAF cells. Binding of an ATPcompetitive inhibitor to one protomer within a RAF dimer results in both abolition of the catalytic activity of the inhibitor-bound RAF and transactivation of the other. Transactivation of RAF homo- and heterodimers is therefore responsible for induction of MEK/ERK phosphorylation by RAF inhibitors in cells with wild-type BRAF. Our model explains the paradoxical phenomenon of ERK activation by RAF inhibitors, previously reported by others ${ }^{20-22}$. Other kinases that exist in dimeric or multimeric complexes may behave in a similar manner. Recently, another model to explain these phenomena has been proposed ${ }^{23}$. That study reports that only selective BRAF inhibitors activate CRAF and ERK signalling, whereas pan-RAF inhibitors do not. Our data that all RAF inhibitors activate ERK signalling at low concentrations, that the phenomenon occurs in BRAF-null cells and that binding to CRAF activates CRAF- and BRAF-dependent ERK signalling render that model unlikely.

Nevertheless, the clinical utility of these inhibitors depends on their inhibition of ERK signalling in tumour cells with BRAF(V600E). Because transactivation of wild-type RAF requires dimerization and depends on RAS activity, we hypothesized that the levels of RAS activity in BRAF(V600E) mutant tumours may not be sufficient to support transactivation. If so, activation of RAS in BRAF(V600E) cells should prevent inhibition of ERK signalling by RAF inhibitors. In $293 \mathrm{H}$ cells overexpressing BRAF(V600E) and in HT29 tumour cells with endogenous BRAF(V600E), ERK signalling was inhibited by either PLX4032/PLX4720 or a MEK inhibitor. In contrast, when mutant RAS was coexpressed with BRAF(V600E) in either cell, ERK signalling became resistant to PLX4032/PLX4720, but remained sensitive to the MEK inhibitor (Fig. 4d and Supplementary Fig. 14a, b).

The data are consistent with the idea that RAF inhibitors suppress ERK signalling in BRAF(V600E) tumours because the level of RAS activation in these cells is insufficient to support transactivation of wild-type RAF and inhibition of BRAF(V600E) activity becomes the dominant

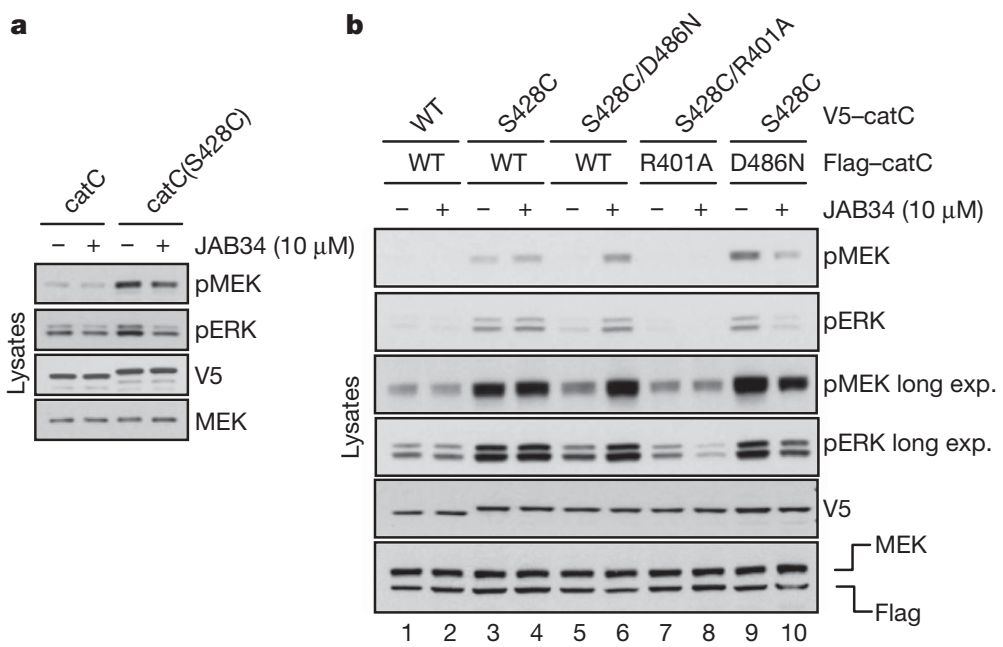

C

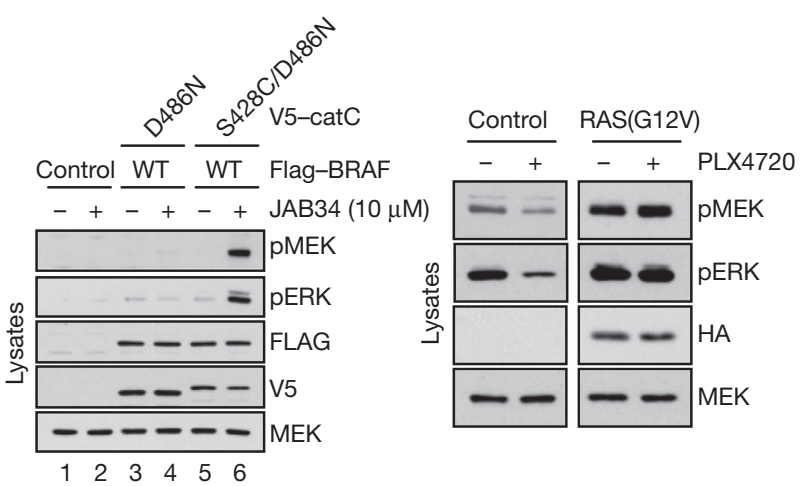

Figure 4 | MEK/ERK activation occurs via transactivation of RAF dimers. a, Similarly to RAF inhibitors, JAB34 inhibits MEK/ERK at higher concentrations. $293 \mathrm{H}$ cells expressing V5-tagged catC or catC(S428C) were treated with either vehicle or $10 \mu \mathrm{M} \mathrm{JAB} 34$ for $1 \mathrm{~h}$. $\mathbf{b}$, Coexpression of drugsensitive V5-tagged catC with drug-resistant Flag-catC reveals that activation in the homodimer occurs in trans. $293 \mathrm{H}$ cells expressing the indicated mutants $\mathrm{V} 5$-tagged cat $\mathrm{C}$ and Flag-tagged catC were treated with a dose of JAB34 $(10 \mu \mathrm{M}$ for $1 \mathrm{~h})$ that inhibits catC(S428C) when expressed alone. c, Activation in the context of the heterodimer BRAF-CRAF occurs in trans. $293 \mathrm{H}$ cells coexpressing Flag-tagged wild-type BRAF and V5-tagged kinase-dead catC ( catC $(\mathrm{D} 486 \mathrm{~N})$ ) (lanes 3,4$)$ or JAB34-sensitive/kinase-dead catC (catC(S428C/D486N)) (lanes 5, 6) treated with vehicle or $10 \mu \mathrm{M} \mathrm{JAB} 34$ for $1 \mathrm{~h}$ are shown. d, HT-29 cells (colorectal; BRAF(V600E)) were transfected with EGFP or HA-tagged NRAS(G12V) and treated with PLX4720 $(1 \mu \mathrm{M}$ for $1 \mathrm{~h})$. Lysates were blotted for pMEK and pERK. 
effect of the drug. The findings indicate that increases in RAS activation or RAF dimerization may be sufficient to cause drug resistance.

The clinical implications of these findings are profound. BRAF(V600E) tumours and some with RAS mutation are dependent on ERK signalling. However, in clinic, a MEK inhibitor had only a $12 \%$ response rate in melanomas with BRAF mutation ${ }^{24}$. MEK inhibitors block ERK signalling in all tumour and normal cells and the dose of the drug that can be administered is limited by toxicity. RAF inhibitors and MEK inhibitors might have been expected to have similar biological effects. Our findings show otherwise. RAF inhibitors will be useful for the treatment of tumours driven by BRAF(V600E), but could have deleterious effects in some contexts due to ERK activation. However, the absence of ERK inhibition in normal cells may allow administration of high doses of RAF inhibitors and thus more complete inhibition of ERK signalling in BRAF(V600E) tumours than is possible with MEK inhibitors.

The recent phase I clinical trial of PLX4032 in metastatic melanoma strikingly confirmed these predictions ${ }^{4,5}$. High serum levels of drug were achieved with modest toxicity and resulted in profound inhibition of ERK signalling in tumours. Tumour regression was observed in more than $90 \%$ of patients with BRAF(V600E) mutation, with $64 \%$ achieving a partial response by RECIST criteria. We believe that the remarkable activity of this drug, compared to that of MEK inhibitors, is due to its ability to inhibit ERK signalling in tumours more completely because of the absence of ERK inhibition in normal tissue.

Resistance to PLX4032 does develop, with a median time to disease progression of 8-9 months ${ }^{5}$. Potential mechanisms include gatekeeper mutations in BRAF and activating mutations in parallel signalling pathways. Our results indicate the possibility of novel mechanisms as well. Lesions that activate RAS or, as recently reported, overexpression of wild-type RAF isoforms ${ }^{25}$ could result in inability of RAF inhibitors to suppress ERK signalling in the tumour and thus lead to resistance.

\section{METHODS SUMMARY}

Compounds and cell culture. PLX4032 and PLX4720 were obtained from Plexxikon. PD0325901 was synthesized in the MSKCC Organic Synthesis Core Facility by O. Ouerfelli. Sorafenib was synthesized using published procedures ${ }^{26}$. JAB13 and JAB34 were synthesized as previously described ${ }^{17}$. All other drugs were obtained from Calbiochem. Drugs were dissolved in DMSO and stored at $-20^{\circ} \mathrm{C}$. Cells were maintained in either DMEM or RPMI, supplemented with $2 \mathrm{mM}$ glutamine, antibiotics and $10 \%$ fetal bovine serum. Wild-type, $\mathrm{Braf}^{-1-}$ and $\mathrm{Craf}^{-1-}$ MEFs were provided by M. Baccarini. 293H cells were from Invitrogen. All other cell lines were from the American Type Culture Collection. Antibodies. Western blot analysis was performed as described ${ }^{1}$. The following antibodies were used: p217/p221-MEK (pMEK), p202/p204-ERK (pERK), p338CRAF, p380-RSK, p573-RSK, MEK, ERK, Myc tag (Cell Signaling), p621-CRAF, V5 tag (Invitrogen), ARAF, BRAF (Santa Cruz Biotechnology), Flag tag (Sigma), CRAF (BD Transduction Laboratories), HA tag (Covance). For immunoprecipitations of tagged proteins, the following reagents were used: anti-V5 agarose affinity gel, antiFlag M2 affinity gel, anti-c-Myc agarose affinity gel (all from Sigma).

Plasmids. Plasmids encoding HA-tagged wild-type and mutant NRAS were obtained from Biomyx. Plasmids for wild-type BRAF and BRAF(V600E) were provided by W. Kolch and were used as template to create Flag-tagged constructs. All other plasmids were created using standard cloning methods, with pcDNA3.1 (Invitrogen) as a vector. Mutations were introduced using the sitedirected Mutagenesis Kit (Stratagene). The catalytic domain of CRAF (catC) was created by truncating the first 305 amino acids of CRAF.

Kinase assays. RAF kinase assays were conducted in the presence of $100 \mu \mathrm{M}$ ATP, at $30^{\circ} \mathrm{C}$ for $20 \mathrm{~min}$. Recombinant inactive K97R MEK (Millipore) was used as a substrate and kinase activity was estimated by immunoblotting for pMEK. Transfections. Cells were seeded on $35 \mathrm{~mm}$ or $100 \mathrm{~mm}$ plates and transfected the following day using Lipofectamine 2000 (Invitrogen).

Full Methods and any associated references are available in the online version of the paper at www.nature.com/nature.

Received 13 November 2009; accepted 16 February 2010.

Published online 23 February 2010.

1. Solit, D. B. et al. BRAF mutation predicts sensitivity to MEK inhibition. Nature 439, 358-362 (2006).
2. McDermott, U. et al. Identification of genotype-correlated sensitivity to selective kinase inhibitors by using high-throughput tumor cell line profiling. Proc. Natl Acad. Sci. USA 104, 19936-19941 (2007).

3. Wellbrock, C. et al. V599EB-RAF is an oncogene in melanocytes. Cancer Res. 64, 2338-2342 (2004).

4. Chapman, P. et al. Early efficacy signal demonstrated in advanced melanoma in a phase I trial of the oncogenic BRAF-selective inhibitor PLX4032. Eur. J. Cancer 7 (suppl.), 5 (2009).

5. Flaherty, K. et al. Phase I study of PLX4032: Proof of concept for V600E BRAF mutation as a therapeutic target in human cancer. J. Clin. Oncol. 27 (suppl.), abstr. 9000 (2009).

6. Tsai, J. et al. Discovery of a selective inhibitor of oncogenic B-Raf kinase with potent antimelanoma activity. Proc. Natl Acad. Sci. USA 105, 3041-3046 (2008)

7. Wellbrock, C., Karasarides, M. \& Marais, R. The RAF proteins take centre stage. Nature Rev. Mol. Cell Biol. 5, 875-885 (2004).

8. Young, A. et al. Ras signaling and therapies. Adv. Cancer Res. 102, 1-17 (2009).

9. Konecny, G. E. et al. Activity of the dual kinase inhibitor lapatinib (GW572016) against HER-2-overexpressing and trastuzumab-treated breast cancer cells. Cancer Res. 66, 1630-1639 (2006).

10. Weber, C. K., Slupsky, J. R., Kalmes, H. A. \& Rapp, U. R. Active Ras induces heterodimerization of CRaf and BRaf. Cancer Res. 61, 3595-3598 (2001).

11. Wan, P. T. et al. Mechanism of activation of the RAF-ERK signaling pathway by oncogenic mutations of B-RAF. Cell 116, 855-867 (2004).

12. Rushworth, L. K., Hindley, A. D., O'Neill, E. \& Kolch, W. Regulation and role of Raf1/B-Raf heterodimerization. Mol. Cell. Biol. 26, 2262-2272 (2006).

13. Cutler, R. E. Jr, Stephens, R. M., Saracino, M. R. \& Morrison, D. K. Autoregulation of the Raf-1 serine/threonine kinase. Proc. Natl Acad. Sci. USA 95, 9214-9219 (1998).

14. Okuzumi, T. et al. Inhibitor hijacking of Akt activation. Nature Chem. Biol. 5, 484-493 (2009).

15. Cameron, A. J., Escribano, C., Saurin, A. T., Kostelecky, B. \& Parker, P. J. PKC maturation is promoted by nucleotide pocket occupation independently of intrinsic kinase activity. Nature Struct. Mol. Biol. 16, 624-630 (2009).

16. Karreth, F. A., DeNicola, G. M., Winter, S. P. \& Tuveson, D. A. C-Raf inhibits MAPK activation and transformation by B-Raf(V600E). Mol. Cell 36, 477-486 (2009).

17. Blair, J. A. et al. Structure-guided development of affinity probes for tyrosine kinases using chemical genetics. Nature Chem. Biol. 3, 229-238 (2007).

18. Sun, Y. et al. Growth inhibition of nasopharyngeal carcinoma cells by EGF receptor tyrosine kinase inhibitors. Anticancer Res. 19, 919-924 (1999).

19. Rajakulendran, T., Sahmi, M., Lefrancois, M., Sicheri, F. \& Therrien, M. A dimerization-dependent mechanism drives RAF catalytic activation. Nature 461, 542-545 (2009)

20. Hall-Jackson, C. A. et al. Paradoxical activation of Raf by a novel Raf inhibitor. Chem. Biol. 6, 559-568 (1999).

21. King, A. J. et al. Demonstration of a genetic therapeutic index for tumors expressing oncogenic BRAF by the kinase inhibitor SB-590885. Cancer Res. 66, 11100-11105 (2006).

22. Hoeflich, K. P. et al. Antitumor efficacy of the novel RAF inhibitor GDC-0879 is predicted by $\mathrm{BRAF}^{\mathrm{V} 600 \mathrm{E}}$ mutational status and sustained extracellular signalregulated kinase/mitogen-activated protein kinase pathway suppression. Cancer Res. 69, 3042-3051 (2009).

23. Heidorn, S. J. et al. Kinase-dead BRAF and oncogenic RAS cooperate to drive tumor progression through CRAF. Cell 140, 209-221 (2010).

24. Dummer, R. et al. AZD6244 (ARRY-142886) vs temozolomide (TMZ) in patients (pts) with advanced melanoma: An open-label, randomized, multicenter, phase II study. J. Clin. Oncol. 26 (suppl.), abstr. 9033 (2008).

25. Montagut, C. et al. Elevated CRAF as a potential mechanism of acquired resistance to BRAF inhibition in melanoma. Cancer Res. 68, 4853-4861 (2008).

26. Bankston, D. et al. A scaleable synthesis of BAY 43-9006: A potent Raf kinase inhibitor for the treatment of cancer. Org. Process Res. Dev. 6, 777-781 (2002).

Supplementary Information is linked to the online version of the paper at www.nature.com/nature.

Acknowledgements $\mathrm{We}$ are grateful to $\mathrm{W}$. Kolch for the BRAF plasmids and M. Baccarini for the RAF knockout MEFs. We thank J. Blair for synthesis of JAB compounds and A. Dar, S. Chandarlapaty and D. Solit for discussions. This work has been funded by the Melanoma Research Alliance, the Starr Cancer Consortium, an $\mathrm{NIH} / \mathrm{NCl}$ P01 grant (1P01CA129243-02) and by Joan's Legacy: United Against Lung Cancer Foundation (P.I.P., N.R.). K.M.S. would like to thank NIH-2R01EB001987, The Children's Tumor Foundation and the Waxman Foundation for funding.

Author Contributions P.I.P. and C.Z. designed research, performed experiments, analysed data and co-wrote the paper. G.B. provided reagents, analysed data and co-wrote the paper. K.M.S. and N.R. designed research, analysed experiments and co-wrote the paper.

Author Information Reprints and permissions information is available at www.nature.com/reprints. The authors declare no competing financial interests. Correspondence and requests for materials should be addressed to N.R. (rosenn@mskcc.org). 


\section{METHODS}

Compounds and cell culture. PLX4032 and PLX4720 were obtained from Plexxikon. PD0325901 was synthesized in the MSKCC Organic Synthesis Core Facility by O. Ouerfelli. Sorafenib was synthesized using published procedures ${ }^{26}$. JAB13 and JAB34 were synthesized as previously described ${ }^{17}$. All other drugs were obtained from Calbiochem. Drugs were dissolved in DMSO to yield $10 \mathrm{mM}$ stock and stored at $-20^{\circ} \mathrm{C}$. Cells were maintained in DMEM (MEFs, $293 \mathrm{H}, \mathrm{NIH} 3 \mathrm{~T} 3$ and HeLa) or RPMI (all other cell lines), supplemented with $2 \mathrm{mM}$ glutamine, antibiotics and $10 \%$ fetal bovine serum. Wild-type, $\mathrm{Braf}^{-1-}$ and $\mathrm{Craf}^{-1-}$ MEFs were provided by M. Baccarini. $293 \mathrm{H}$ cells were from Invitrogen. All other cell lines were from the American Type Culture Collection.

Antibodies. Western blot analysis was performed as described ${ }^{1}$. The following antibodies were used: p217/p221-MEK (pMEK), p202/p204-ERK (pERK), p338-CRAF, p380-RSK, p573-RSK, MEK, ERK, Myc tag (Cell Signaling), p621-CRAF, V5 tag (Invitrogen), ARAF, BRAF (Santa Cruz Biotechnology), Flag tag (Sigma), CRAF (BD Transduction Laboratories), HA tag (Covance). For immunoprecipitations of tagged proteins, the following reagents were used: anti-V5 agarose affinity gel, anti-Flag M2 affinity gel, anti-c-Myc agarose affinity gel (all from Sigma).
Plasmids. Plasmids encoding HA-tagged wild-type and mutant NRAS were obtained from Biomyx. Plasmids expressing Myc-tagged wild-type BRAF and $\mathrm{BRAF}(\mathrm{V} 600 \mathrm{E})$ were provided by $\mathrm{W}$. Kolch and were used as templates to create Flag-tagged constructs. All other plasmids were created using standard cloning methods, with pcDNA3.1 (Invitrogen) as a vector. Mutations were introduced using the site-directed Mutagenesis Kit (Stratagene). The catalytic domain of CRAF (catC) was created by truncating the first 305 amino acids of CRAF.

Immunoprecipitations and kinase assays. Cells were lysed in lysis buffer ( $50 \mathrm{mM}$ Tris, pH 7.5, 1\% NP40, $150 \mathrm{mM} \mathrm{NaCl}, 10 \%$ glycerol, $1 \mathrm{mM}$ EDTA) supplemented with protease and phosphatase inhibitor cocktail tablets (Roche). Immunoprecipitations were carried out at $4{ }^{\circ} \mathrm{C}$ for $4 \mathrm{~h}$, followed by three washes with lysis buffer and, in cases of subsequent kinase assay, one extra wash with kinase buffer ( $25 \mathrm{mM}$ Tris, $\left.\mathrm{pH} 7.5,10 \mathrm{mM} \mathrm{MgCl}_{2}\right)$. RAF kinase assays were conducted in the presence of $100 \mu \mathrm{M} \mathrm{ATP}$, at $30^{\circ} \mathrm{C}$ for $20 \mathrm{~min}$. Recombinant inactive K97R MEK (Millipore) was used as a substrate and the reaction was terminated with the addition of sample buffer and boiling. Kinase activity was estimated by immunoblotting for pMEK.

Transfections. Cells were seeded on $35 \mathrm{~mm}$ or $100 \mathrm{~mm}$ plates and transfected the following day using Lipofectamine 2000 (Invitrogen). Twenty-four hours later, cells were collected for subsequent analysis. 